\title{
ON EXTENDED QUASIFUCHSIAN GROUPS
}

\author{
Bernard Maskit
}

In this paper, we complete our investigation of finitely generated Kleinian groups that preserve two disjoint open sets. In what follows, $G$ is a finitely generated Kleinian group. It was shown in [M1] that if $G$ has two invariant components (of the set of discontinuity), then there is a quasiconformal homeomorphism $w$, mapping the extended complex plane, $\hat{\mathbf{C}}$ onto itself, where $w$ conjugates $G$ onto a Fuchsian group (of the first kind). Other proofs of this were given by Kra and Maskit [KM1], and by Marden [Ma1] and Thurston [T] in the torsion-free case. This result was then extended by Kra and Maskit [KM2] who showed that if $G$ has exactly two components, $U$ and $L$, then there is a quasiconformal homeomorphism $w: \hat{\mathbf{C}} \rightarrow \hat{\mathbf{C}}$, where $w G w^{-1}$ is again Kleinian, and $w$ maps $U$ onto the upper half-plane, and $L$ onto the lower half-plane. Further work in this direction appears in [M4, p. 316], where it was shown that if $G$ preserves a disc, then $G$ is a quasiconformal deformation of a Fuchsian group, perhaps of the second kind.

An extended quasifuchsian group is a finitely generated Kleinian group, $\tilde{G}$, with the following property. There are two disjoint, connected, non-empty, open sets, $U$ and $L$, both contained in the set of discontinuity $\Omega=\Omega(\tilde{G})$, where $\bar{U} \cup \bar{L}=$ $\hat{\mathbf{C}}$, and $U \cup L$ is $\tilde{G}$-invariant. We require further that $\partial U \cap \Omega(\tilde{G})=\partial L \cap \Omega(\tilde{G})$ be a disjoint union of countably many topological arcs. In the special case that $U$ and $L$ are the upper and lower half-planes, $\mathbf{U}$ and $\mathbf{L}$, respectively, then we say that $\tilde{\mathbf{G}}$ is an extended Fuchsian group.

An extended quasifuchsian group $\tilde{\mathbf{G}}$ contains a subgroup, $G$, called the core, which preserves both $U$ and $L$. It could be that $G=\tilde{G}$; if not, then $G$ is a subgroup of index 2 .

Observe that the sets $U$ and $L$ are necessarily simply connected. An extended quasifuchsian group is of the first kind if $\partial U=\partial L$ consists only of limit points; it is of the second kind otherwise.

Throughout this paper, we will use the word circle to refer to a Euclidean circle on $\hat{\mathbf{C}}$. A topological mapping of a circle into $\hat{\mathbf{C}}$ will be referred to as a Jordan curve.

We will assume, without further mention, that all Kleinian groups are finitely generated. We will also assume, unless specifically stated otherwise, that all homeomorphisms are orientation preserving.

Research supported in part by National Science Foundation Grant no. DMS 8701774. The author also wishes to thank the Mathematical Sciences Research Institute for its hospitality.

doi:10.5186/aasfm.1990.1523 
We say that the Kleinian group, $\tilde{G}$, is a topological deformation of the the Kleinian group, $\tilde{F}$, if there is a homeomorphism $w: \hat{\mathbf{C}} \rightarrow \hat{\mathbf{C}}$, so that $w \tilde{G} w^{-1}=\tilde{F}$. For the special case that $\tilde{G}$ is extended quasifuchsian, and $\tilde{F}$ is extended Fuchsian, we also require that $w(U)=\mathbf{U}$, and $w(L)=\mathbf{L}$.

Theorem 1. Let $G$ be an extended quasifuchsian group. Then there is an extended Fuchsian group $\tilde{F}$, so that $\tilde{G}$ is a topological deformation of $\tilde{F}$.

In order to prove this theorem, we first classify the extended quasifuchsian groups of the second kind in terms of their function group signatures; this appears as Theorem 2 .

Corollary 1. Let $\tilde{G}$ be a finitely generated Kleinian group which preserves a Jordan curve. Then $\tilde{G}$ is a topological deformation of an extended Fuchsian group.

Since we can approximate $\partial U \cap \Omega(G)$ by smooth arcs, we obtain the following.

Corollary 2. Let $G$ be a finitely generated Kleinian group which preserves the Jordan curve, $\gamma$. Then there is a $G$-invariant curve $\gamma^{\prime}$, arbitrarily close to $\gamma$, there is an extended Fuchsian group $F$, and there is a quasiconformal homeomorphism $w: \hat{\mathbf{C}} \rightarrow \hat{\mathbf{C}}$, where $w G w^{-1}=F$, and $w\left(\gamma^{\prime}\right)$ is a circle.

By adjoining reflection in the real axis, and then restricting appropriately, one can go from an extended Fuchsian group to a discrete group of isometries (including orientation reversing ones) of the hyperbolic plane. Conversely, if one has a group of isometries of the hyperbolic plane, then one can adjoin reflection in the real axis, and then restrict to the orientation preserving half, and so obtain an extended Fuchsian group. These remarks, together with Theorem 1, reduce the topological classification of the class of extended quasifuchsian groups to the (known) topological classification of the class of discrete groups of isometries of the hyperbolic plane. This result is needed for the topological classification of the panelled groups introduced in [M3], where particular examples were constructed; this classification will be carried out elsewhere.

Theorem 3. The topological classification of the class of finitely generated extended quasifuchsian groups is coextensive with the topological classification of the class of finitely generated discrete groups of isometries, including orientation reversing ones, of the hyperbolic plane.

The proof of this theorem appears in Section 5 .

1. We now assume that $\tilde{G}$ is an extended quasifuchsian group of the second kind. We denote $\partial U=\partial L$ by $\gamma$; this is a countable union of arcs, called boundary arcs, together with limit points of $\tilde{G}$. We consistently orient the boundary arcs so that $U$ lies on the left as they are traversed in the positive direction. The sets $U$ and $L$ are both connected, and $G$-invariant, hence simply connected. 
In general, if $A$ is a boundary arc, then, since the core, $G$, is finitely generated, $\operatorname{Stab}_{G}(A)$ is loxodromic (including hyperbolic) cyclic, and $A$ is precisely invariant under $\operatorname{Stab}_{G}(A)$. The only exceptions to this rule occur if $G$ is elementary; if $G$ is finite cyclic, then all of $\gamma$ is a $G$-invariant boundary arc; if $G$ is parabolic cyclic, then all of $\gamma$ except for the parabolic fixed point is a $G$-invariant boundary arc.

For future reference, we note that since $U$ and $L$ are simply connected, the boundary arcs are naturally ordered.

Since $G$ is of the second kind, $\tilde{G}$, qua Kleinian group, has only one component, necessarily invariant. Since $G$ is a deformation of a geometrically finite group, it is also geometrically finite. Hence $\tilde{G}$ is a geometrically finite function group.

2. We next explore the function group signature $\sigma=(K, t)$ of $\tilde{G}$ (see [M4, Chapter X]; we will follow the notation of [M4] throughout). We know that the limit set, $\Lambda=\Lambda(\tilde{G})=\Lambda(G)$ is a Cantor set; since the structure subgroups are stabilizers of connected components of $\Lambda$, every part of $K$ is elementary.

Since Fuchsian groups of the second kind are all obtained from cyclic groups using combination theorems, the same is true of quasifuchsian groups of the second kind. We can restate this in terms of signatures.

Proposition 1 [M4, p. 315]. Let $G$ be a quasifuchsian group of the second kind. Then either $G$ is a Schottky group (i.e., $K$ contains just one part of signature $(0,0))$, or $\sigma$ has the following two properties:

(i) every part has signature $(0,2 ; \alpha, \alpha), 2 \leq \alpha \leq \infty$; and

(ii) $K$ contains no connectors.

The converse to the above is also true $[\mathrm{M} 4, \mathrm{p} .316]$; that is, if the signature of the function group $G$ satisfies the above properties, then $G$ is quasifuchsian of the second kind.

Theorem 2. Let $\tilde{G}$ be a geometrically finite function group of signature $\sigma=(K, t)$. If $\tilde{G}$ is an extended quasifuchsian group, then the following hold.

(A) Every part has basic signature $(0,0)$, or $(0,2 ; \alpha, \alpha)$ or $(0,3 ; 2,2, \alpha), 2 \leq \alpha$ $\leq \infty$.

(B) Every connector is a 2-connector.

(C) For every part $P$, at most two special points of $P$ are endpoints of connectors.

Proof. We have already remarked that every part in the signature of $\tilde{G}$ is elementary; an equivalent statement is that every structure subgroup is elementary. We also know that every structure subgroup of $G$ is cyclic. Since the structure subgroups are maximal subgroups with simply connected invariant components, the intersection of $G$ with a $\tilde{G}$-structure subgroup is a $G$-structure subgroup. Hence every structure subgroup of $\tilde{G}$ is either cyclic, or a $\mathbf{Z}_{2}$-extension of a cyclic group. One easily checks the list of elementary groups with at most one limit point to see that every structure subgroup of $\tilde{G}$ is either trivial, elliptic or parabolic cyclic, dihedral, or infinite dihedral. This is condition (A). 
We next assume that there are parts $P$ and $P^{\prime}$, not necessarily distinct, and that there is an $\alpha$-connector between $P$ and $P^{\prime}, \alpha>2$. We know [M4, p. 281] that a special point on a part of signature $(0,2 ; \beta, \beta)$ can only be connected to the other special point on that part, and then only when $\beta<\infty$; also, two parts of signature $(0,3 ; 2,2, \infty)$ cannot have an $\infty$-connector between them. Hence the only possibilities for there to be an $\alpha$-connector in $K, \alpha>2$, is that we have a part $P$ with signature $(0,2 ; \alpha, \alpha), 2<\alpha<\infty$, with a connector having both endpoints on $P$, or that we have two parts $P$ and $P^{\prime}$, of signature $(0,3 ; 2,2, \alpha)$, $2<\alpha<\infty$, and there is an $\alpha$-connector between them.

In the first case, there is a corresponding subgroup of $\tilde{G}$ generated by an elliptic element $a$, of order $\alpha$, and a loxodromic element, $b$, where $a$ and $b$ commute. Since the fixed points of $b$ lie on $\Lambda(G)$, so do those of $a$. Since a either preserves or interchanges $U$ and $L$, it has order 2 . In the second case, the resulting group is double dihedral, where again there is an elliptic element of order $\alpha$ commuting with a loxodromic element. This shows the necessity of (B).

In view of statement (B), the only case we need consider for $(\mathrm{C})$ is that $P$ has basic signature $(0,3 ; 2,2,2)$. Let $H$ be a structure subgroup lying over $P$; then $H$ is isomorphic to the Klein 4 -group. Since $G$ is a free product of cyclic groups, $H$ is not a subgroup of $G$. Let $J=H \cap G$; then $J$ is cyclic of order 2 . Since $J$ preserves the topological discs, $U$ and $L$, it has a fixed point in each of them; these fixed points necessarily lie in $\Omega(G)=\Omega(\tilde{G})$. An equivalent statement is that the special point on $P$ corresponding to the fixed points of $J$ is not the endpoint of a connector. $\square$

Easy modifications of the proof of Theorem 1, given below, show that the above conditions are also sufficient. However, in general, the function group signature does not suffice to determine the extended quasifuchsian group; that is, in general, a function group whose signature satisfies the above properties can be made into an extended quasifuchsian group of the second kind in infinitely many essentially distinct ways.

3. Our next goal is to understand the relationship between the boundary arcs and the structure loops.

Let ${ }^{\circ} \Omega$ be $\Omega(\tilde{G})$ with all fixed points of elliptic transformations removed. Similarly, let ${ }^{\circ} \tilde{S}={ }^{\circ} / \Omega \tilde{G}$. Let $\left\{w_{1}, \ldots, w_{k}\right\}$ be a set of dividers on ${ }^{\circ} \tilde{S}$; this is a set of simple disjoint loops with the property that each $w_{m}$, when raised to an appropriate power, lifts to a loop in ${ }^{\circ} \tilde{\Omega}$, and, if we adjoin small loops about the special points of $\tilde{S}$, then the covering $p:{ }^{\circ} \tilde{\Omega} \rightarrow{ }^{\circ} \tilde{S}$ is the highest regular covering of ${ }^{\circ} \tilde{S}$ for which these loops, when raised to these powers, lift to loops; we also require that this set of loops be minimal. (Since every structure subgroup of $\tilde{G}$ is elementary, $\tilde{G}$ contains no accidental parabolic transformations.) The connected components of the preimages of the dividers in ${ }^{\circ} \tilde{\Omega}$ are the structure loops; each structure loop is kept precisely invariant either by the identity or by a maximal elliptic cyclic subgroup. One can reformulate property (B) as saying that each 
divider either lifts to a loop in ${ }^{\circ} \tilde{\Omega}$, or its square does; equivalently, the stabilizer of each structure loop is either trivial or cyclic of order 2 .

Since $U$ and $L$ are simply connected, no structure loop can lie entirely in either $U$ or $L$; i.e., every structure loop crosses a boundary arc.

The following is a version of the planarity theorem; the proof is a variant of Gromov's proof (see [M4, p. $251 \mathrm{ff}]$.$) .$

Lemma 1. For an extended quasifuchsian group of the second kind, the dividers can be chosen so that every structure loop intersects the set of boundary arcs at exactly two points.

Proof. It is essentially obvious that $\pi_{1}(\Omega)$ is generated by loops having exactly two points of intersection with boundary arcs, and it is clear that we can choose these loops to be geodesics in the Poincaré metric of ${ }^{\circ} \tilde{\Omega}$. Let $\hat{A}$ be the set of geodesics on ${ }^{\circ} \tilde{\Omega}$ having exactly two crossings of boundary arcs, and let $A$ be the set of projections of loops in $\hat{A}$ to ${ }^{\circ} \tilde{S}$.

Let $w_{1}$ be the shortest loop in $A$. We first observe that $w_{1}$ is simple, or a power of a simple loop. If not, then there are two distinct geodesics, $W$ and $W^{\prime}$, in $\hat{A}$, both lying over $w_{1}$, and having one, and hence two, points of intersection; call these points of intersection $x$ and $y$. Then we can write $W=U \cdot V, W^{\prime}=U^{\prime} \cdot V^{\prime}$, where, except for direction, all four arcs go between $x$ and $y$. Since there is no shorter geodesic than $W$ (or $W^{\prime}$ ) with exactly two crossings of boundary arcs, no one of these four arcs can be shorter than the other three. Then one of the four loops $U^{-1} \cdot U^{\prime}, U \cdot V^{\prime}, V \cdot U^{\prime}$, or $V^{-1} \cdot V^{\prime}$ has the same length as $W$. Of course the corresponding geodesic is shorter. This is a contradiction, for all four of these loops have exactly two points of intersection with boundary arcs. Hence $w_{1}$ is a simple loop which, when raised to some power (necessarily 1 or 2 ), lifts to a loop in ${ }^{\circ} \tilde{\Omega}$.

Let $N_{1}$ be the normal closure of the homotopy class of $w_{1}$ in $\pi_{1}\left({ }^{\circ} \tilde{S}\right)$, together with appropriate powers of small loops about special points, and let $N$ be the defining subgroup of the covering $p:{ }^{\circ} \tilde{\Omega} \rightarrow{ }^{\circ} \tilde{S}$; then $N_{1} \subset N$. If $N_{1} \neq N$, choose a loop $\hat{w}$ whose homotopy class is in $N$, but not in $N_{1}$, where $\hat{w}$ has only finitely many crossings of boundary arcs. Then $\hat{w}$ is freely homotopic to a product of loops which do not cross $w_{1}$ (see [M4, p. 252]). Then at least one of these loops also lies in $N$, but not in $N_{1}$.

Now we can assume that $\hat{w}$ is a loop whose homotopy class lies in $N$, but not $N_{1}$, and that $\hat{w}$ is disjoint from $w_{1}$. However, a lift $\hat{W}$ of $\hat{w}$ might have several crossings of boundary arcs. It is clear that we can find two successive points of intersection of $\hat{W}$ with $\gamma=\partial U$, call them $x$ and $y$, where $x$ and $y$ are successive points of intersection on both $\hat{w}$ and on $\gamma$; where we are using the natural ordering of the boundary arcs. For the sake of argument, we can assume that the arc of $\hat{W}$ between $x$ and $y$ that contains no other points of intersection with $\gamma$ lies in $L$. Find points, $x^{\prime}$ and $y^{\prime}$, on $\hat{W}$ near $x$ and $y$, respectively, where $x^{\prime}$ and $y^{\prime}$ both 
lie in $U$. Connect $x^{\prime}$ to $y^{\prime}$ by an arc in $U$ that does not intersect $\hat{W}$. We have now split $\hat{W}$ into a product of two loops, one has exactly two points of intersection with $\gamma$, and the other has two fewer points of intersection than does $\hat{W}$. Hence we can also assume that $\hat{W}$ has exactly two points of intersection with boundary arcs.

Now define the new class, $A_{1}$, of projections to ${ }^{\circ} \tilde{S}$ of geodesics having exactly two points of intersection with boundary arcs, and disjoint from all lifts of $w_{1}$. We know that $A_{1} \neq \emptyset$; let $w_{2}$ be the projection of the shortest geodesic in $A_{1}$; then $w_{2}$ is simple, disjoint from $w_{1}$, and its homotopy class lies in $N-N_{1}$.

Define $N_{2}$ to be the normal closure of $w_{1}$ and $w_{2}$, together with appropriate powers of small loops about special points. If $N_{2}=N$, we are finished; if not, then find $w_{3}$ as above; etc. Since ${ }^{\circ} \tilde{S}$ is topologically finite, this process ends after finitely many steps.

4. We are now in a position to prove Theorem 1 . As already remarked, the theorem was proven for $\tilde{G}$ of the first kind in [M1] and [KM2]; hence we need only consider the case that $\tilde{G}$ is of the second kind. After some preliminary observations, the proof is essentially an application of combination theorems. The basic idea is to write $\tilde{G}$ as a repeated amalgamated free product, HNN-extension, and free product. Then, using the fact that the structure loops all have exactly two points of intersection with boundary arcs, reproduce the construction to obtain an extended Fuchsian group, $\tilde{F}$. This construction also yields fundamental domains that are homeomorphic. Then, using the actions of the groups, we obtain a homeomorphism between $\Omega(\tilde{G})$ and $\Omega(\tilde{F})$. The desired result then follows from the following.

Extension theorem [M2]. Let $F$ and $G$ be geometrically finite function groups, and let $w: \Omega(F) \rightarrow \Omega(G)$ be a homeomorphism that induces a typepreserving isomorphism of $F$ onto $G$. Then $w$ is the restriction of a global homeomorphism; in particular, $G$ is a topological deformation of $F$.

We start our proof with the basic groups; these are the elementary groups with at most one limit point.

Lemma 2. If $\tilde{G}$ is a basic group (i.e., $\sigma=(K, 0)$, where $K$ has exactly one elementary part and no connectors), then $\tilde{G}$ is a topological deformation of an extended Fuchsian group.

Proof. If $\tilde{G}$ is finite, then it is either cyclic or (finite) dihedral. Also, if $\tilde{G}$ has one limit point, then it is either parabolic cyclic, or infinite dihedral. We first construct the extended Fuchsian models, to be named $\tilde{F}$, for these groups.

In general, for the cyclic group of order $p$, we normalize so that the fixed points of $\tilde{F}$ are at $\pm i$, and we let $\tilde{E}$ be the outside of the isometric circles; this is a fundamental domain. There is also one special case of a cyclic group of order 2 , where the non-trivial element interchanges the half-planes. In this case, we choose 
$\tilde{F}$ to be generated by $z \rightarrow 1 / z$, and we let the unit disc be the fundamental domain, $\tilde{E}$.

The extended Fuchsian groups with basic signature $(0,3 ; 2,2, \alpha), 2 \leq \alpha<\infty$ can be normalized so as to be generated by an element $a$ of order $\alpha$, having its fixed points at $\pm i$, and an element $b$ of order 2 , having its fixed points on the real axis. Normalize so that $\infty$ is not a fixed point of any element of order 2 , and so that the fixed points of elements of order 2 lie in the interval $[-d, d]$, with $\pm d$ being fixed points; of course, $d$ depends on $\alpha$. Then the Ford region, $\tilde{E}$, has just two sides; one passes through $\pm i$ and $+d$, the other passes through $\pm i$ and $-d$.

For the parabolic cyclic group, we choose the generator to be $z \rightarrow z+2$, and we let the fundamental domain $\tilde{E}=\{z|| \operatorname{Re}(z) \mid<1\}$.

The extended Fuchsian group with basic signature $(0,3 ; 2,2, \infty)$ can be normalized to be generated by $z \rightarrow z+2$, and $z \rightarrow-z+1$. We choose the set $\tilde{E}=\left\{z|| \operatorname{Re}(z) \mid<\frac{1}{2}\right\}$ as a fundamental domain.

If $\tilde{G}$ is finite cyclic, and every element preserves $U$ and $L$, then choose a geometric generator $g$ for $\tilde{G}$, and choose any point $x$ on $\gamma$. Connect $x$, and all its $\tilde{G}$ translates, to the fixed points of $\tilde{G}$ by hyperbolic geodesics in both $U$ and $L$. These form arcs connecting the fixed points, and these arcs separate $\hat{\mathbf{C}}$ into regions. Any one of these regions will serve as a fundamental domain, $\tilde{D}$; note that $g$ identifies the two sides of $\tilde{G}$, and that $\gamma$ intersects each of the sides in exactly one point. It is clear that one can map $\tilde{D}$ homeomorphically onto $\tilde{\mathbf{E}}$, preserving the identifications of the sides, and mapping the directed arc, $\gamma \cap \tilde{D}$, onto the directed arc of the real axis in $\tilde{\mathbf{E}}$; with this choice, $U$ is mapped onto U. Now extend using the actions of $\tilde{G}$ and $\tilde{F}$.

If $\tilde{G}$ is cyclic of order 2 , where the non-trivial element interchanges $U$ and $L$, then draw the geodesics in $U$ and $L$ between the fixed points. These divide $\hat{\mathbf{C}}$ into two regions; either one will serve as a fundamental domain. It is easy to map this fundamental domain homeomorphically onto the unit disc, while mapping the directed arc $\gamma \cap \tilde{D}$ onto the appropriate directed arc of the real axis.

If $\tilde{G}$ is dihedral, then the core $G$ is finite cyclic. There are $|G|$ half-turns that interchange $U$ and $L$, so they have their fixed points on $\gamma$. Draw hyperbolic lines from the fixed points of $G$ to the fixed points of these half-turns. These arcs divide $\hat{\mathbf{C}}$ into regions; any one of them will serve as a fundamental domain for $\tilde{G}$; pick one and call it $\tilde{D}$. Let $\tilde{F}$, with fundamental domain $\tilde{E}$, be the $|G|$-dihedral group normalized as above. It is clear that one can map $\tilde{D}$ homeomorphically onto $\tilde{E}$ so that the map is equivariant on the boundary; that is, the identifications of $\tilde{G}$ on the boundary of $\tilde{D}$ are conjugated into the identifications of $\tilde{F}$ on the boundary of $\tilde{E}$, and we can choose this map so that the directed arc $\gamma \cap \tilde{D}$ is mapped onto the directed arc of the real axis inside $\tilde{E}$. The desired result now follows by using the actions of $\tilde{G}$ and $\tilde{F}$.

If $\tilde{G}$ is parabolic cyclic, then choose any point $x$ on $\gamma$ other than the fixed point of $\tilde{G}$. Connect $x$, and all its translates, to the fixed point of $\tilde{G}$ by hyperbolic 
geodesics in both $U$ and $L$. These form Jordan curves that divide $\hat{\mathbf{C}}$ into regions. Pick one of these regions, call it $\tilde{D}$, and observe that it is a fundamental domain for $\tilde{G}$. Now map it homeomorphically onto the fundamental domain $\tilde{E}$ for the parabolic cyclic Fuchsian group, $\tilde{F}$, so that the identifications on the boundary are preserved, and so that the directed arc of intersection of $\gamma$ with $\tilde{D}$ is mapped onto the directed arc of the real axis in $\tilde{E}$. This yields a homeomorphism of $\Omega(\tilde{G})$ onto $\Omega(\tilde{F})$; now apply the extension theorem.

If $\tilde{G}$ is infinite dihedral, then its core $G$ is parabolic cyclic. Exactly as above, draw hyperbolic lines in $U$ and $L$ from the fixed point of $G$ to the fixed points of the half-turns in $\tilde{G}$, and let $\tilde{D}$ be one of the regions cut out by these curves. Let $\tilde{F}$, with fundamental domain $\tilde{E}$, be the corresponding infinite dihedral group acting on $\mathbf{U}$ and $\mathbf{L}$. As above, map $\tilde{D}$ onto $\tilde{E}$ preserving the identifications of the sides, and mapping the directed arc of $\gamma \cap \tilde{D}$ onto the directed arc of the real axis in $\tilde{E}$. Then extend by the actions of $\tilde{G}$ and $\tilde{F}$, and use the extension theorem. $\square$

Lemma 3. If $K$ is connected, and $t=0$, then $\tilde{G}$ is a topological deformation of an extended Fuchsian group.

Proof. If there are no connectors, then there is only one part $P$, which either has signature $(0,2 ; \alpha, \alpha)$, or $(0,3 ; 2,2, \alpha)$; i.e., $\tilde{G}$ is either parabolic or elliptic cyclic, or dihedral, including infinite dihedral. These cases are all covered by Lemma 2.

We next consider the special case that $K$ contains exactly one part $P$ of signature $(0,2 ; 2,2)$, and there is a connector between the two special points. In this case $\tilde{G}$ has the form $\mathbf{Z}+\mathbf{Z}_{2}$, so $G$ is loxodromic cyclic. The corresponding extended Fuchsian group can be normalized so as to be generated by $z \rightarrow 2 z$ and $z \rightarrow-z$; choose the quadrilateral $\tilde{E}=\{1<|z|<2\} \cap\{\operatorname{Re}(z)>0\}$ as a fundamental domain.

Let $g$ be a generator of $G$, and let $h \in \tilde{G}$ be an elliptic element of order 2 . The fixed points of $G$ divide $\gamma$ into two arcs $C_{1}$ and $C_{2}$. Choose $C_{1}$ to be the arc going in the positive direction from the repelling fixed point of $g$. Choose a point $x$ on $C_{1}$, and let $y=g(x)$. Use the Poincaré metric in both $U$ and $L$ to construct the orthogonals to the axis of $g$ from both $x$ and $y$. These orthogonals form two arcs which, together with the arcs of the axis of $g$ they cut off, form a quadrilateral, $\tilde{D}$, which is a fundamental domain for $\tilde{G}$. Map $\tilde{D}$ onto $\tilde{E}$, preserving the identifications of the sides, and mapping $\tilde{D} \cap \gamma$, traversed positively, onto the corresponding arc, traversed positively, of the real axis in $\tilde{E}$. Then extend using the actions of the groups to a homeomorphism of $\Omega(\tilde{G})$ onto $\Omega(\tilde{F})$; then use the extension theorem.

For the general case, where $K$ is connected and $t=0$, write the parts as $P_{1}, \ldots, P_{n}$, where $P_{m}$ has signature $\left(0,3 ; 2,2, \alpha_{m}\right)$, and there is a 2 -connector between $P_{m}$ and $P_{m+1}$. There may or may not also be a 2 -connector between 
$P_{n}$ and $P_{1}$; if there is, then the parts form a loop; otherwise, they form a chain. If there is only one part $P$, with a connector both starting and ending at $P$, then we say that the parts form a loop with $n=1$.

We first take up the case that the parts form a chain. If $n=1$, then there is only one part and no connectors; the desired result is given in Lemma 2. Let $H_{1}$ be a structure subgroup lying over $P_{1}$; let $H_{2}$ be a structure subgroup lying over $P_{2}$, where $H_{1} \cap H_{2}$ has order 2 , etc. Let $\tilde{G}_{n-1}$ be the subgroup of $\tilde{G}$ generated by $H_{1}, \ldots, H_{n-1}$. We can assume that there is a topological deformation, $\varphi_{n-1}$ of $\tilde{G}_{n-1}$ onto an extended Fuchsian group, $\tilde{F}_{n-1}$, which maps $U$ onto $\mathbf{U}$. There is also a topological deformation, $\varphi_{n}$, of $H_{n}$ onto an extended Fuchsian group, $M_{n}$; we need to renormalize $\varphi_{n}$ and $M_{n}$.

There is a structure loop $W$ that separates the fixed points of $\tilde{G}_{n-1}$ from those of $H_{n}$; the stabilizer, $\tilde{J}$, of $W$ has order 2 . One of the topological discs bounded by $W$ is kept precisely invariant by $\tilde{J}$ in $\tilde{G}_{n-1}$; call it $C$. Then $\varphi_{n-1}(C)$ is a topological disc kept precisely invariant by a subgroup $\tilde{I}$ of $\tilde{F}_{n-1}$. Let $\hat{C}$ be a circle inside $\varphi_{n-1}(C)$, where $\hat{C}$ is orthogonal to the real axis and $\hat{C}$ bounds a precisely invariant disc. Now normalize $M_{n}$ so that it still preserves the upper and lower half-planes, so that $\tilde{I} \subset M_{n}$, so that one of the fixed points of $\tilde{I}$ lies on the boundary of the fundamental domain for $M_{n}$, and so that the other disc bounded by $\hat{C}$ is precisely invariant under $\tilde{I}$ in $M_{n}$. Then by the first combination theorem [M4, p. $149 \mathrm{ff}$.], $\tilde{F}_{n}=\left\langle\tilde{F}_{n-1}, M_{n}\right\rangle$ is Kleinian, and of course it preserves the real axis.

Since $\varphi_{n-1}$ and $\varphi_{n}$ are both orientation preserving, and both map $U$ onto $\mathbf{U}$, they both map $W$ onto curves with the same orientation, and these curves both cross the real axis at exactly two points. It follows that we can deform $\varphi_{n-1}$ so that it maps $W$ onto $\hat{C}$, while still mapping $U$ onto $\mathbf{U}$, and mapping the disc precisely invariant under $\tilde{J}$ in $\tilde{G}_{n-1}$ onto the corresponding disc precisely invariant under $\tilde{I}$ in $\tilde{F}_{n-1}$. Similarly, we can deform $\varphi_{n}$ so that it also maps $W$ onto $\hat{C}$, while mapping $U$ onto $\mathbf{U}$, and mapping the disc precisely invariant under $\tilde{J}$ in $H_{n}$ onto the corresponding disc precisely invariant under $\tilde{I}$ in $M_{n}$. Since both maps have the same orientation on $W$, and they both homeomorphically map $W$ onto $\hat{C}$, we can deform $\varphi_{n}$ in a neighborhood of $W$, so that the two maps agree on $W$. It is standard (see [M4, p. $299 \mathrm{ff}$.]) to replace $\varphi_{n-1}$ and $\varphi_{n}$ by a single homeomorphism, $\psi_{n}: \Omega\left(\tilde{G}_{n}\right) \rightarrow \Omega\left(\tilde{F}_{n}\right)$, where $\psi_{n}$ conjugates $\tilde{G}_{n}$ onto $\tilde{H}_{n}$, while mapping $U$ onto $\mathrm{U}$. Then, by the extension theorem, $\psi_{n}$ can be extended to a topological deformation.

The last case to be considered is that the $n$ parts form a loop. In this case, there is an additional connector between the other 2-point on $P_{1}$, and the other 2-point on $P_{n}$. Let $\tilde{G}_{n}$ and $\tilde{F}_{n}$ be as above. Then there is a topological deformation $\psi_{n}$ from $\tilde{G}_{n}$ onto $\tilde{F}_{n}$, where $\psi_{n}(U)=\mathbf{U}$. There are also structure loops $W_{1}$ and $W_{2}$ in $\Omega\left(\tilde{G}_{n}\right)$, each having exactly two points of intersection with boundary arcs, and each bounding a disc that is precisely invariant under the 
appropriate cyclic subgroup of order 2 . There is also an element $\tilde{g} \in \tilde{G}$ mapping the interior of the disc bounded by $W_{1}$ onto the exterior of the disc bounded by $W_{2}$. There are two possibilities: either $\tilde{g}$ preserves both $U$ and $L$, or $\tilde{g}$ interchanges them; if necessary, we compose $\tilde{g}$ with one of the elliptics of order 2 , so that $\tilde{g}$ preserves both $U$ and $L$. We take the images of these two discs under $\psi_{n}$, and draw small precisely invariant circles $\hat{C}_{1}$ and $\hat{C}_{2}$ inside them, where $\hat{C}_{1}$ and $\hat{C}_{2}$ are both orthogonal to the real axis. Since $W_{1}$ and $W_{2}$ each has exactly two crossings with boundary arcs, we can deform $\psi_{n}$ so that it maps $W_{m}$ onto $\hat{C}_{m}$, while still mapping $U$ onto $U$. Now choose an element $f_{m}$, mapping the inside of $\hat{C}_{1}$ onto the outside of $\hat{C}_{2}$, and preserving $\mathbf{U}$ and $\mathbf{L}$. It is automatic that $f \circ i_{1} \circ f_{-1}=i_{2}$, where $i_{m}$ generates $\operatorname{Stab}\left(\hat{C}_{m}\right)$. Now deform $\psi_{n}$ further near $\hat{C}_{1}$ so that $\psi_{n} \circ \tilde{g}\left|W_{1}=f \circ \psi_{n}\right| W_{1}$. It is now straightforward (see [M4, p. $299 \mathrm{ff}]$.$) to restrict \psi_{n}$ to an appropriate fundamental domain for $\tilde{G}=\left\langle\tilde{G}_{n}, \tilde{g}\right\rangle$, and extend using the group actions, to obtain a homeomorphism of $\Omega(\tilde{G})$ onto $\Omega(\tilde{F})$. Again, the extension theorem shows that this has an extension to $\hat{C}$. $\square$

Lemma 3 yields Theorem 1 in the case that there are no 1 -dividers; i.e., $K$ is connected, and $t=0$. We now come to the induction step on the number of 1-dividers.

We first consider the case that every 1 -divider is dividing (then $t=0$ ). Let $W$ be a 1 -structure loop, and let $A$ be the set of translates of $W$. Let $R_{1}$ and $R_{2}$ be the regions of the complement of $A$ on either side of $W$. Let $\tilde{G}_{m}=\operatorname{Stab}\left(R_{m}\right)$. Since the projection of $W$ divides $S, \tilde{G}_{1}$ and $\tilde{G}_{2}$ are not conjugate in $\tilde{G}$. Also, as in [M4, p. $285 \mathrm{ff}$.], the signature $\sigma_{m}$ of $\tilde{G}_{m}$ has the form $\sigma_{m}=\left(K_{m}, 0\right)$, where $K_{\tilde{m}}$ is a disjoint union of connected components of $K$, with $K_{1}+K_{2}=K$. Let $\tilde{F}_{m}$ be an extended Fuchsian group which is a topological deformation of $\tilde{G}_{m}$. Let $\varphi_{m}$ be the global homeomorphism conjugating $\tilde{G}_{m}$ onto $\tilde{F}_{m}$. Looking at $\tilde{G}_{m}$, $W$ bounds a disc $C_{m}$ that is precisely invariant under the identity. Let $\hat{G}_{m}$ be a circular disc inside the topological disc $\varphi_{m}\left(C_{m}\right)$, where $\hat{C}_{m}$ is orthogonal to the real axis. Now normalize $\tilde{F}_{1}$ and $\tilde{F}_{2}$ so that $\hat{C}=\hat{C}_{1}=\hat{C}_{2}$, and so that the two precisely invariant discs are on opposite sides of $\hat{C}$. Since $W$ intersects $\gamma$ at exactly two points, we can deform $\varphi_{m}$ so that it maps $W$ onto $\hat{C}$, while still mapping $U$ onto $\mathbf{U}$. We further deform $\varphi_{1}$, still mapping $U$ onto $\mathbf{U}$, so that $\varphi_{1}\left|W=\varphi_{2}\right| W$. Finally, as above, we define a single homeomorphism $\varphi$ on an appropriate fundamental domain for $\tilde{G}$, then extend to $\Omega(\tilde{G})$ using the actions of $\tilde{G}$ and $\tilde{F}=\left\langle\tilde{F}_{1}, \tilde{F}_{2}\right\rangle$, and finally extend to the limit set using the extension theorem.

In the final case, let $W_{1}$ be a 1 -structure loop whose projection is a nondividing 1-divider. As above, let $A$ be the set of translates of $W_{1}$. Let $R_{1}$ and $R_{2}$ be the regions cut out by $A$ on either side of $W_{1}$, and let $H_{m}=\operatorname{Stab}\left(W_{m}\right)$. In this case, there is a transformation $\tilde{g} \in \tilde{G}$ mapping $R_{1}$ onto $R_{2} ;$ let $W_{2}=g\left(W_{1}\right)$, $R=R_{2}$, and $\tilde{G}_{0}=H_{2}$. There are exactly two possibilities: either $\tilde{g}$ preserves both $U$ and $L$, or it interchanges them. It was shown in [M4, p. $285 \mathrm{ff}$.] that 
the signature of $\tilde{G}_{0}$ is $(K, t-1)$. Hence we can assume that there is an extended Fuchsian group $\tilde{F}_{0}$, and there is a topological deformation $\varphi$ of $\tilde{G}_{0}$ onto $\tilde{F}_{0}$, where $\varphi(U)=\mathbf{U}$. We note that $W_{1}$ and $W_{2}$ bound discs that are precisely invariant under the identity in $\tilde{G}_{0}$. Let $\hat{C}_{1}$ and $\hat{C}_{2}$ be circles, where $\hat{C}_{m}$ lies in the image under $\varphi$ of the precisely invariant disc bounded by $W_{m}$, and where $\hat{C}_{m}$ is orthogonal to the real axis. Since $W_{1}$ and $W_{2}$ each intersects $\gamma$ at exactly two points, we can deform $\varphi$ so that it maps $W_{m}$ onto $\hat{C}_{m}$, while still mapping $U$ onto U. Find a transformation $f$ mapping the outside of $\hat{C}_{1}$ onto the inside of $\hat{C}_{2}$, and either preserving $\mathbf{U}$ and $\mathbf{L}$, or interchanging them, according as $\tilde{g}$ preserves $U$ and $L$, or interchanges them. Then deform $\varphi$ further so that $f \circ \varphi\left|W_{1}=\varphi \circ \tilde{g}\right| W_{1}$, and proceed as above. $\square$

5. We now turn to the proof of Theorem 3. Let $G$ be an extended quasifuchsian group, with core $G_{0}$, and let $g$ be some element of $G$ interchanging the upper and lower regions. We saw above that $G$ is a deformation of an extended Fuchsian group, $F$. We normalize $F$ so that it acts on the real axis, and we denote reflection in the real axis by $*$. Let $\tilde{F}=\langle F, *\rangle$, the group generated by $F$ and *, and let $\hat{F}$ be the subgroup of $\tilde{F}$ that preserves $\mathbf{H}^{2}$. Then $\hat{F}$ is a discrete group of isometries of $\mathbf{H}^{2}$.

We need to show that the topological type of $\mathbf{H}^{2} / \hat{F}$ is determined by $G$. To this end, it suffices to assume that $G$ and $F$ are extended Fuchsian groups, where $F$ is a deformation of $G$. That is, there is a a homeomorphism $\varphi: \hat{\mathbf{C}} \rightarrow \hat{\mathbf{C}}$, where $\varphi$ preserves the upper half-plane, and conjugates $G$ onto $F$. Let $\tilde{G}(\tilde{F})$ be the group $\langle G, *\rangle(\langle F, *\rangle)$, and let $\hat{G}(\hat{F})$ be the $\mathbf{H}^{2}$ preserving half of $\tilde{G}(\tilde{F})$.

Let $\psi={ }^{*} \circ \varphi \circ^{*} \circ \varphi^{-1}$; note that its restriction to $\mathbf{H}^{2}$ is an orientation preserving homeomorphism which commutes with every element of $F$. Hence, by the Bers-Greenberg theorem [BG] (see also Marden [Ma]), $\psi \mid \mathbf{H}^{2}$ is invariantly isotopic to the identity in the complement of the fixed points of the elliptic elements of $F$. Equivalently, $*$ and $\varphi \circ * \circ \varphi^{-1}$ are invariantly isotopic as maps from the complement of the elliptic fixed points of $F$ in the lower half-plane to the complement of the elliptic fixed points of $F$ in the upper half-plane. It follows that if $f \in F$ interchanges the upper and lower half-planes, then $f \circ *$ and $f \circ \varphi \circ * \circ \varphi^{-1}$ project to homotopic maps of $\mathbf{H}^{2}$ modulo the orientation preserving half of $\hat{F}$, from which it follows that $\mathbf{H}^{2} / \hat{F}$ and $\mathrm{H}^{2} / \varphi \hat{F} \varphi^{-1}$ are homeomorphic.

Conversely, if $\hat{F}$ is a finitely generated discrete group of isometries of $\mathbf{H}^{2}$, then set $\tilde{F}=\langle\hat{F}, *\rangle$, and let $F$ be the orientation preserving half of $\tilde{F}$. Observe that $\tilde{F}$ is an extended Fuchsian group. $\square$

\section{References}

[BG] Bers, L., and L. Greenberg: Isomorphisms between Teichmüller spaces. - Advances in the theory of Riemann surfaces, Annals of Mathematical Studies 66. Princeton University Press, Princeton, N.J., year, 53-79.

[KM1] KrA, I., and B. MAskit: Involutions on Kleinian groups. - Bull. Amer. Math. Soc. 78, $1972,801-805$. 
[KM2] KRA, I., and B. MASKIT: Bases for quadratic differentials. - Comment. Math. Helv. 57, $1982,603-626$.

[Ma] Marden, A.: On homotopic mappings of Riemann surfaces. - Ann. of Math. 90, 1969, 1-8.

[Ma1] Marden, A.: The geometry of finitely generated Kleinian groups. - Ann. of Math. 99, 1974, 383-462.

[M1] MASkit, B.: On boundaries of Teichmüller spaces and on kleinian groups II. - Ann. of Math. 91, 1970, 607-639.

[M2] Maskit, B.: On the classification of Kleinian groups: II. Signatures. - Acta Math. 138, 1977, 17-42.

[M3] Maskit, B.: Panelled web groups. - Kleinian Groups and Related Topics. (Proceedings of the Conference held in Oaxtapec, 1981). Lecture Notes in Mathematics 971. SpringerVerlag, Berlin-Heidelberg-New York, 1983, 79-108.

[M4] Maskit, B.: Kleinian groups. - Springer-Verlag, Berlin-New York-Heidelberg, 1988.

[T] Thurston, W.: The geometry and topology of 3-manifolds. - Lecture notes, Department of Mathematics, Princeton University, Princeton, NJ, 1977.

State University of New York at Stony Brook

Department of Mathematics

Stony Brook, NY 11794-3651

U.S.A.

Received 10 January 1989 